\title{
Author Index Vol. 209, 1995
}

\section{Abe, T. 92}

Adachi-Usami, E. 101 Addabbo,G. 228 Akazawa,K. 199 Akiya,S. 96 Aktan,S.G. 132

Ambach,W. 329 Angeletti,G. 88 Antoniazzi, E. 315 Aspiotis, M. 68 Ayaki, M. 37

Baerveldt, G. 275 Bandello,F. 149 Bardot,J. 136 Battaglia Parodi, M. 106, 248 Bellini, G. 187 Bellone,A. 117 Berros,P. 223 Best, J.A. van 7 Blumthaler, M. 329 Bocin,E. 187 Bolla,N. 117 Bondel,E. 248 Brancato,R. 149 Bredehorn, T. 79 Buchberger, W. 84 Bujger,Z. 242

Calabria, G. 177 Casellato,L. 297 Caselli,E. 340 Cassai,E. 340 Castagna, I. 53,267 Cennamo, G. 17 Cerovski,B. 242 Charpentier, D. 223 Ciftci,O.U. 270 Cortesini, L. 1 Cosenzi, A. 187

Daicker,B. 47 Daniele,C. 88,217 Daniele,S. 88,217 Daxecker, F. 329 DeBaets,F. 40 Delft, J.L. van 7 DelPrete,A. 17 Demircan, N. 336 Denis, D. 136 Denis, P. 223 Devin,F. 306

Domenici-Lombardo, L. 1 Duncker, G.I.W. 25,79

Ekert,M. 242

Falk,M. 60 Famà,F. 53,267 Faussone-Pellegrini, M.S. 1 Ferreri, G. 267 Ferri,C. 217 Flammer, J. 11,129,302 Fujio,N. 292 Fujishima, H. 208 Fujitani, A. 220 Fukisawa, K. 199 Fukui,K. 292 Furukawa, H. 96 Furukawa, Y. 37

Gabrielian, K. 212 Gaspar, A.Z. 11 Gasser,P. 11 Genovese, F.R. 267 Girard,N.J. 284 Girard,P. 323 Gracner, B. 14

Haciyakupoglu, G. 225, 336

Haciyakupoglu, S. 225

Hamada, T. 96

Hashimoto, T. 299

Hatano,H. 155

Hayasaka, S. 172,220,251,256,289

Heilmann, P. 56

Hendrickson, P. 302

Hiroi,K. 299

Hojs, R. 14

Honda, Y. 299

Hong, Y J. 319

Iester, M. 177 Igarashi,H. 292 Igarashi,S. 292 Incorvaia, C. 340 Inomata,H. 44, 199 Ishibashi,T. 44 Ishikura,H. 172 Isobe,T. 155

Jourdan, T. 306

Kaiser, HJ. 129,302 Kalogeropoulos, Ch. 64

Kaluza, G. 122 Karampatakis, V. 194 Karpouzas, I. 323 Kashii,S. 346 Kaya,M. 336 Kieselbach, G.F. 60 Kim,H.B. 319 Kim,S.J. 319 Kimura,H. 212,346 Kimura,T. 101 Kitsos,G. 68 Kobayashi,S. 172 Koda,R.P. 212 Koide,O. 96 Kondo,M. 172 Kondo,T. 113,160 Kremmer, S. 70,233 Kremser,B.G. 60 Kubota,T. 44 Küchle,M. 145 Kurihara, K. 44 Kurihashi, K. 27 Kwon,O.W. 319 
Lafaut,B.A. 40 Lattanzio,R. 149 Lautenschläger, Ch. 56 Lebreuil,G. 284 Leccisotti, A. 182,228 Lee,M.B. 275 Lee,S.Y. 319 Leino, M. 21 Li,H.K. 260 Lietz,A. 129 Lohmann, W. 7 Longhini, L. 340 Lucciani, A. 306

Maestranzi, G. 149 Manservigi, R. 340 Mansour, A.M. 260 Marone, P. 315 Masai, H. 346

Masami, P. 113 Maserati, R. 315 Matsumoto, C.S. 280 Matsuo,K. 280,331 Matsuo,N. 109

Matsuo,T. 109 Matsuura, T. 331 Maumenee, I.H. 136 Meire, F. 40 Mencucci, R. 1

Menicacci, F. 228 Mermoud, A. 275 Minami, H. 203 Minckler, D.S. 275 Miyake, M. 299

Monini,P. 340 Monzen, Y. 280 Monzillo,V. 315 Moseley, MJ. 52 Moser, M. 84 Motolese, E. 228

Nakagawa, H. 109

Nakajima, N. 256 Nakatsu,A. 113, 160 Nakatsuka,K. 280 Nakatuka,K. 331 Nguyen, N.X. 145

Ninomiya, H. 96 Noda, S. 289 Nolle, B. 25 Nose,Y. 199

Ogino,N. 203 Oguz,M. 225 Ohnishi-Nio, T. 203 Ohno,S. 155 Okinami, S. 203 Onozu,H. 251

Othman,I. 336 Özbilgin, M.K. 336 Özdemir,N. 225 Ozdemir,O. 270

Pahor,D. 14 Parmeggiani, F. 340 Paschides, C.A. 141 Pastena, B. 17 Paterra,N. 228 Perversi, L. 315 Pillunat, L.E. 70 Polat,S. 336 Polito,E. 182,228

Protti,R. 117 Proust, H. 284 Psilas,K. 64,68, 141

Ramovecchi, P. 106 Rao,N.A. 275 Ravalico,G. 106,187,248 Raybaud,CA. 284 Reha Ersöz, T. 225 Remky,H. 190 Ridings, B. 284 Rieger,G. 84 Rinaldi,G. 187 Riss,J.M. 284 Rolando, M. 177 Rosa,N. 17 Rouhiainen, H. 21 Ryan,SJ. 212

Sacerdote, A. 187 Sakamoto, T. 212 Salmeri, G. 53 Salvi,G. 1 Santamaria, S. 267 Saracco, J.-B. 136,306 Schepens, C.L. 88 Schmiederer, M. 79 Schönherr,U. 145 Sebastiani, A. 17 Serhath,M. 132 Shiao,S. 155 Shibuya,Y. 172 Shimazaki,J. 96,208 Solimano,N. 187 Souza,O.F. de 212 Spee,C. 212 Stangos,N. 194 Stefaniotou, M. 64, 141 Stodtmeister, R. 70,233 Stolwijk, T.R· 7 Strempel, I. 122 Stümpfig,D. 129,302 Sugahara,S. 96

Sugai,S. 199 Suzuki, T. 96

Tachi-Ogawa, N. 203 Takahashi,H. 96 Tamai, M. 92 Tanihara, H. 203 Teräsvirta, M. 21 Toda,I. 208

Tolksdorf-Kremmer, A. 70, 233 Topouzis, F. 194 Tost,F. 56 Touameur, S. 223 Traverso, C.E. 177 Tsironi, E. 68 Tsubota,K. 96,208 Turaçh, M.E. 132

Valli,A. 117 Verbraeken, H. 165 Vianna, R.N.G. 40 Volot,F. 136 Voutas,S. 194

Wada,Y. 160 Watanabe, A. 251 Wiegand, W. 7 Wilhelmus, K.R. 278 Winkler,R. 84

Yagi,Y. 208 Yamada,N. 251 Yamamoto, S. 251 Yamashita,K. 172 Yang,H.-Y. 208 Yatsuka,H. 280 Yokoyama, N. 37 Yokoyama, S. 331 Yoshida,A. 292 Yoshida,T. 101 Yoshimura, M. 96 Z. Zingirian

M. 177

Author Index Vol. 209

1995 